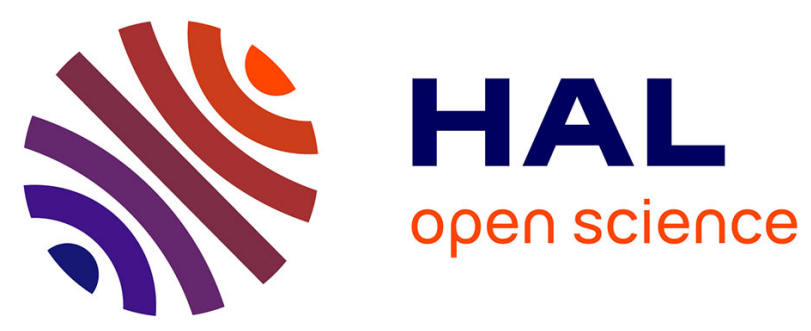

\title{
A COMPARATIVE STUDY OF DERAMPING TECHNIQUES FOR SENTINEL-1 TOPS IN THE CONTEXT OF INTERFEROMETRY
}

Roland Akiki, Raphaël Grandin, Carlo de Franchis, Gabriele Facciolo, Jean-Michel Morel

\section{To cite this version:}

Roland Akiki, Raphaël Grandin, Carlo de Franchis, Gabriele Facciolo, Jean-Michel Morel. A COMPARATIVE STUDY OF DERAMPING TECHNIQUES FOR SENTINEL-1 TOPS IN THE CONTEXT OF INTERFEROMETRY. 2021. hal-03189167

\section{HAL Id: hal-03189167 https://hal.science/hal-03189167}

Preprint submitted on 2 Apr 2021

HAL is a multi-disciplinary open access archive for the deposit and dissemination of scientific research documents, whether they are published or not. The documents may come from teaching and research institutions in France or abroad, or from public or private research centers.
L'archive ouverte pluridisciplinaire HAL, est destinée au dépôt et à la diffusion de documents scientifiques de niveau recherche, publiés ou non, émanant des établissements d'enseignement et de recherche français ou étrangers, des laboratoires publics ou privés. 


\title{
A COMPARATIVE STUDY OF DERAMPING TECHNIQUES FOR SENTINEL-1 TOPS IN THE CONTEXT OF INTERFEROMETRY
}

\author{
Roland Akiki $^{1,3} \quad$ Raphaël Grandin ${ }^{2}$ Carlo de Franchis ${ }^{1,3}$ Gabriele Facciolo $^{1}$ Jean-Michel Morel $^{1}$ \\ ${ }^{1}$ Université Paris-Saclay, CNRS, ENS Paris-Saclay, Centre Borelli, France \\ ${ }^{2}$ Institut de Physique du Globe de Paris - Université Paris VII - FRANCE \\ ${ }^{3}$ Kayrros SAS
}

\begin{abstract}
In this study, we compare the different spectral centering methods (referred to as deramping) for the Sentinel-1 images acquired with the TOPSAR method, in the context of interferometry. The deramping is a necessary step prior to image interpolation. We show the analogy between two different approaches in the literature and propose our own improvements. The proposed deramping method approximately re-centers the spectrum, i.e. a small residual spectral shift remains. We validate our improvements with experiments on Sentinel-1 data, and show that interpolating the images containing this residual spectral shift should not induce considerable errors.
\end{abstract}

Index Terms - Deramping, Demodulation, TOPSAR, Sentinel-1, Spectral centering

\section{INTRODUCTION}

SAR (synthetic aperture radar) images have become an important data source in the remote sensing community, especially because of their ability to acquire images independently of the time of day and the weather conditions. Their usefulness has increased especially with the emergence of some advanced applications like interferometry. Interferometery consists in having at least two complex SAR images acquired at two different dates for the same geographical areas. The image is written as $I_{1}=A_{1} \exp \left(j \Phi_{1}\right)$ where $I_{1}, A_{1}, \Phi_{1}$ refer to the image signal, the ground reflectivity and the phase difference between the sent and received wave respectively. The same can be written for $I_{2}$. The phase difference between the two images is computed with

$$
\operatorname{Interf}\left(I_{1}, I_{2}\right)=I_{1} \cdot I_{2}^{\star}=A_{1} A_{2} \exp \left(j\left(\Phi_{1}-\Phi_{2}\right)\right) \text {. }
$$

$\Phi_{1}-\Phi_{2}$ in (1) contains valuable information about the change in time of flight from the ground scatterers to the sensor on the satellite platform.

Work partly financed by IDEX Paris-Saclay IDI 2016, ANR-11-IDEX0003-02, Office of Naval research grant N00014-17-1-2552 and N00014-20S-B001, DGA Astrid project « filmer la Terre » $\mathrm{n}^{\circ}$ ANR-17-ASTR-0013-01, MENRT, and by a grant from ANRT.
This difference in time of flight can be attributed to many factors, one of which is the ground deformation between the two dates. Therefore, interferometry is of interest for many applications, including but not limited to natural disasters (earthquakes, volcanoes) or ground motion by man-made activities like hydraulic fracturing for oil and gas extraction.

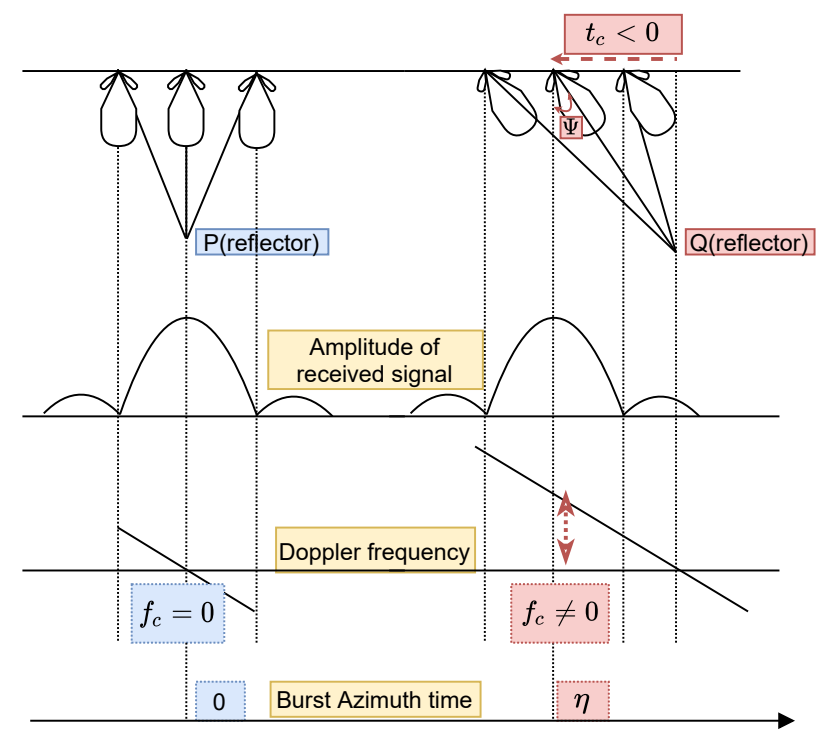

Fig. 1: Downward view of the slant range plane during a TOPSAR burst acquisition. On the left, in blue, the antenna beam is orthogonal to the trajectory (near the middle of the burst). The Doppler frequency at the point of maximum amplitude is $0 \mathrm{~Hz}$. On the right, in red, at azimuth time $\eta$, the beam has a squint angle $\Psi$ w.r.t. the zero Doppler plane. The point of max amplitude is reached earlier, and the Doppler frequency at this position is not null and is proportional to the radial velocity component (projection on line of sight)

Improving our ability to generate interferograms is therefore a subject of great interest. We focus our study on the Sentinel-1 satellite because of its high revisit time (6 days) and freely available data.

The preferred acquisition of a SAR image for interferome- 
try using Sentinel-1 is in the Interferometric Wide (IW) swath mode. Using the TOPSAR (Terrain Observation with Progressive Scans SAR) technique, during the burst acquisition, the antenna beam is steered "continuously" in the azimuth direction with a steering rate of $k_{\Psi}$ as seen in Figure 11 This introduces a linear squint $\Psi$ with azimuth time $\eta$. The steering of the antenna beam will of course modify the Doppler properties of the signal. For example, in Figure 1, the Doppler frequency of point $\mathrm{Q}$ is linearly dependent on $\eta$ and $f_{c}(\eta) \neq 0$ since $\Psi(\eta) \neq 0$. This phenomenon thus induces a linear frequency shift with the azimuth time in the spectrum of the focused data.

When dealing with the problem of interferometry, one usually has a primary acquisition and a set of secondary acquisitions. The secondary acquisitions need to be coregistered to the primary coordinate system, thus the data needs to be interpolated. However, special care should be taken when resampling this complex signal that has a linearly shifted spectrum. The correction that needs to be applied prior to interpolation is called "Deramping".

Our contributions are:

- Detailing the mathematical equations of a deramping method [1], and showing the analogy with another method of the literature [2];

- Showing the pros and cons of each deramping method, and proposing our own modifications.

\section{RELATED WORK}

Deramping is extensively explained in the ESA (European Space Agency) guidelines [3], where the reader is provided with the necessary tools to perform the deramping and the demodulation operations from Sentinel-1 metadata. First, define $\eta$ as the azimuth time referenced to the center of the burst, $\tau$ as the range time and $k_{t}(\tau)$ as the Doppler rate in the focused data

$$
k_{t}(\tau)=\frac{k_{a}(\tau) k_{s}}{k_{a}(\tau)-k_{s}}=\frac{k_{s}}{\alpha(\tau)}, \quad \alpha(\tau)=1-\frac{k_{s}}{k_{a}(\tau)},
$$

where $k_{a}(\tau)$ is the classical azimuth $\mathrm{FM}$ rate. $k_{s}$ is the Doppler rate induced by the steering of the beam

$$
k_{s} \approx \frac{2 v_{s}}{\lambda} k_{\psi}
$$

where $v_{s}$ is the satellite velocity and $\lambda$ the carrier wavelength and $k_{\psi}$ the rate of rotation in $\mathrm{rad} / \mathrm{s}$.

We also need to define $\eta_{\text {ref }}(\tau)=\eta_{c}(\tau)-\eta_{c}\left(\frac{N s_{\text {swath }}}{2}\right)$ as the azimuth reference time, where $N s_{\text {swath }}$ is the time corresponding to the number of samples in range and $\eta_{c}(\tau)=-\frac{f_{\eta c}(\tau)}{k_{a}(\tau)}$ with $f_{\eta_{c}}(\tau)$ the mean Doppler centroid frequency over the burst. $f_{\eta_{c}}(\tau)$ and $k_{a}(\tau)$ are given as polynomials w.r.t $\tau$ in the metadata.
The deramping function is finally defined as the multiplication of the data, burst by burst, with

$$
\phi(\eta, \tau)=\exp \left(-j \pi k_{t}(\tau)\left(\eta-\eta_{r e f}(\tau)\right)^{2}\right)
$$

After the deramping operation is performed, the spectrum will still be shifted by $f_{\eta_{c}}(\tau)$. Therefore, a demodulation operation is needed to shift the spectrum to baseband. If we wish to perform the demodulation with the deramping, the updated spectral centering function is

$$
\begin{aligned}
\phi(\eta, \tau)=\exp ( & -j \pi k_{t}(\tau)\left(\eta-\eta_{\text {ref }}(\tau)\right)^{2} \\
& \left.-j 2 \pi f_{\eta c}(\tau)\left(\eta-\eta_{\text {ref }}(\tau)\right)\right) .
\end{aligned}
$$

Reramping/remodulation correspond to the application of the complex conjugate of the functions defined in Equations 4 or 5 .

In [2], a detailed study of the complete processing chain for Sentinel-1 interferogram formation is provided. The authors distinguish between two different options for the complex burst interpolation. One of the options consists in modulating the interpolation kernel with the Doppler shift. This option is not going to be considered for our study for the sake of simplicity, since it requires an implementation of interpolation kernels. The second option consists in applying the centering function of Equation 5. Then the data is resampled with a real valued kernel. The reramping function is also resampled before applying it, i.e. if $T$ is the forward transform such that $\left(\eta^{\prime}, \tau^{\prime}\right)=T(\eta, \tau)$, then for every position in the new grid $\left(\eta^{\prime}, \tau^{\prime}\right)$, the original position $(\eta, \tau)=T^{-1}\left(\eta^{\prime}, \tau^{\prime}\right)$ is estimated and inserted in the complex conjugate of Equation 5 . The need for deramping is justified by the fact that a realvalued interpolation kernel acts as a low-pass filter. Therefore, it is necessary to bring the signal to baseband before applying the real-valued kernel. The need for reramping is justified by the fact that deramping is an operation that is performed to re-center the spectrum prior to interpolation, but it biases the interferometric phase, and should therefore be compensated. Indeed, interferometry is performed with the two SAR images in their original frequency band, provided that the coregistration error between the two images is sufficiently small.

The study conducted in [1] shows that it is possible to apply an appropriate deramping function on a secondary acquisition burst prior to resampling and omit the step of reramping. This only works if a matching deramping function has also been applied in the primary acquisition. This method is only relevant in the context of interferometry. Since this method is the one implemented in the interferometric NSBAS software, we were able to carefully reexamine the equations of [1]. The details of this method, along with our own modifications are reported in the next section. 


\section{METHOD}

For the sake of simplicity, we denote the method exposed in [3] and [2] as "EsaDeramping", the one of [1] as "NsbasDeramping". First, we will present "NsbasDeramping", then we will introduce some modifications, that will refer to in the following sections by "Ours". As mentioned previously, "NsbasDeramping" is specific for interferometry. Suppose we have a primary acquisition on which $\mathrm{N}$ secondary acquisitions need to be coregistered. Even though the primary burst will not be resampled, the essence of the method lies in multiplying it with the deramping function defined as

$$
\phi\left(\eta^{(p)}, \tau^{(p)}\right)=\exp \left(-j \pi k_{t}^{(p)}\left(\tau^{(p)}\right)\left(\eta^{(p)}\right)^{2}\right),
$$

where the superscript ${ }^{(p)}$ stands for primary.

Then, for the corresponding burst in a secondary acquisition, the deramping function applied should be matched to the deramping value of the primary, i.e. the same ground feature at coordinates $\left(\eta^{(p)}, \tau^{(p)}\right)$ and $\left(\eta^{(s)}, \tau^{(s)}\right)$ for the primary and secondary bursts respectively should have the same deramping factor applied. Recall that $\eta$ is referenced to the burst middle time and is defined in

$$
\eta^{(p)}=t_{0}^{(p)}+\frac{y_{R}^{(p)}}{P R F}-t_{\text {start }}^{(p)}-\frac{l p b-1}{2 P R F},
$$

for the primary acquisition, where $t_{0}^{(p)}$ refers to the azimuth time of the first line in the image, $y_{R}^{(p)}$ is the azimuth coordinate of the reflector, $P R F$ is the pulse repetition frequency and $t_{\text {start }}^{(p)}$ is the time of the first line in the burst and $l p b$ is the number of lines per burst. The same equation is valid for the secondary acquisition.

It is then possible to estimate the lag in azimuth time $\eta$ between the location of ground the feature in the secondary and the primary burst as

$$
\eta_{l a g}=\eta^{(s)}-\eta^{(p)}=\eta_{\text {off }}+\eta_{\text {shift }},
$$

where $\eta_{o f f}=\frac{y_{R}^{(s)}-y_{R}^{(p)}}{P R F}$ is computed from the transform of the registration and $\eta_{\text {shift }}=t_{0}^{(s)}-t_{0}^{(p)}-\left(t_{\text {start }}^{(s)}-t_{\text {start }}^{(p)}\right)$ is computed from the timing information in the metadata. We can also proceed in the same manner for the range time $\tau^{(p)}=\frac{x_{R}^{(p)}}{F_{r}}+\tau_{0}^{(p)}$, where $x_{R}^{(p)}$ is the range coordinate of the reflector in the primary burst, $F_{r}$ is the range sampling frequency, $\tau_{0}^{(p)}$ is the time of the first range sample in the image. $\tau^{(s)}$ has the same equation as $\tau^{(p)}$. Thus we can find, $\partial \tau=\tau^{(s)}-\tau^{(p)}$. Using a simple approximation for $k_{a} \approx \frac{-2 v_{e f f}^{2}}{\lambda R}$, where $R=\frac{c \tau}{2}$ is the range expressed in $(\mathrm{m})$ and $c$ the light speed in $(\mathrm{m} / \mathrm{s})$, and $v_{\text {eff }}$ is the effective velocity defined from $k_{a}$, we combine with Equation 2 and 3 and get

$$
\alpha=1+\frac{v s}{v_{e f f}^{2}} k_{\Psi} R \approx 1+\frac{k_{\Psi} R}{v_{e f f}}
$$

$$
\partial k_{t}=-\frac{k_{s} \partial \alpha}{\alpha^{2}}=-\frac{k_{s} k_{\Psi} c \partial \tau}{2 v_{e f f}\left(1+\frac{k_{\Psi} R}{v_{e f f}}\right)^{2}}
$$

Finally, using $\partial k_{t}=k_{t}^{(s)}\left(\tau^{(s)}\right)-k_{t}^{(p)}\left(\tau^{(p)}\right)$, the deramping function that multiplies the secondary acquisition at $\left(\eta^{(s)}, \tau^{(s)}\right)$ is defined by

$$
\begin{array}{r}
\phi\left(\eta^{(s)}, \tau^{(s)}\right)=\exp \left\{-j \pi\left(k_{t}^{(s)}\left(\tau^{(s)}\right)-\partial k_{t}\right)\right. \\
\left.\cdot\left(\eta^{(s)}-\eta_{\text {lag }}\right)^{2}\right\} .
\end{array}
$$

Afterwards, the secondary acquisition burst can be resampled.

When looking at Equation 11, it is easy to see how a clear analogy can be made with the method "EsaDeramping" since the deramping function applied at the primary burst in Equation 6 acts similarly to the reramping step. In other words, "NsbasDeramping" is actually starting with the reramping on a regular grid in the primary burst coordinate system (the conjugate of the reramping function is applied). Then, this reramping function is "resampled" to the secondary burst coordinate system and applied. Finally, the secondary burst is resampled.

With this analogy, it is clear that we can improve the deramping applied to the secondary acquisition, especially the part related to $\partial k_{t}$. Instead of using (9), we can simply do the following: for each pixel in the secondary burst, use the registration's transform $T$ to obtain the subpixel position in the primary burst $\left(\eta^{(p)}, \tau^{(p)}\right)=T\left(\eta^{(s)}, \tau^{(s)}\right)$ and plug these values in (6). This method will be henceforth called "Ours".

For "NsbasDeramping" and "Ours", If we have N secondary acquisitions, we perform the deramping once per image. Taking into account the deramping performed at the primary acquisition, we would have in total $\mathrm{N}+1$ deramping operations. Comparing with "EsaDeramping", for each secondary acquisition, we need to deramp and then reramp. Hence, we would have $2 * \mathrm{~N}$ de/re-ramping operations. The computational gain of "NsbasDeramping" and "Ours" comes mainly from the fact that there is a single deramping operation applied in the primary image that replaces all the rerampings of the secondary acquisitions.

Their disadvantage stems from the fact that the phase in the deramping applied to the secondary acquisition in $(10)$ is different from the one specified in $(5)$ by

$$
\begin{aligned}
\Delta \Phi= & -\pi k_{t}(\tau)\left(\eta_{\text {ref }}(\tau)-\eta_{\text {lag }}\right)\left(2 \eta-\eta_{\text {lag }}-\eta_{\text {ref }}(\tau)\right) \\
& +\pi \partial k_{t}\left(\eta-\eta_{\text {lag }}\right)^{2}+2 \pi f_{\eta_{c}}(\tau)\left(\eta-\eta_{\text {ref }}(\tau)\right),
\end{aligned}
$$

where the superscript ${ }^{(s)}$ has been dropped for simplicity. The burst spectrum in azimuth will not be exactly in baseband prior to resampling, and the shift will be equal to $f_{\text {shift }}$ as

$$
\begin{aligned}
f_{\text {shift }}=\frac{1}{2 \pi} \frac{\partial \Delta \Phi}{\partial \eta}= & -k_{t}(\tau)\left(\eta_{\text {ref }}(\tau)-\eta_{\text {lag }}\right) \\
& +\partial k_{t}\left(\eta-\eta_{\text {lag }}\right)+f_{\eta_{c}}(\tau)
\end{aligned}
$$




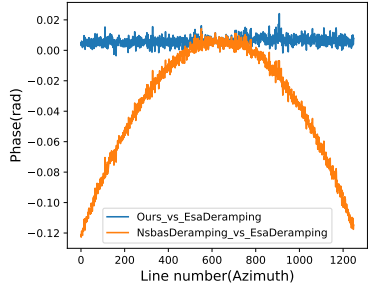

(a) The interferometric phase difference profile

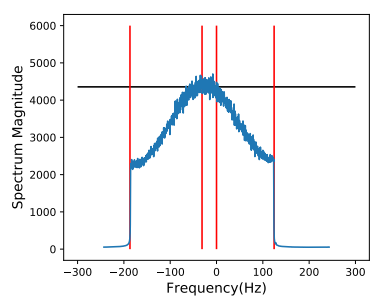

(b) The spectral shift of "Ours"
Fig. 2: The disadvantages of "NsbasDeramping" and "Ours". On the left, "NsbasDeramping" introduces a quadratic phase along the azimuth. On the right, both methods feature a spectral shift prior to resampling

\section{EXPERIMENTS}

The dataset used in the experiments consists of 32 Sentinel1 images acquired over Albania $\left(18.82^{\circ} \mathrm{E} ; 41.02^{\circ} \mathrm{N}\right)$ from 20190803 to 20200205 on the $73^{\text {rd }}$ relative orbit. We set the first image as the primary acquisition, and restricted the study on the second burst of the third subswath. The first test conducted consisted in taking the second image of the dataset as a secondary acquisition. The phase difference profile along the azimuth between "EsaDeramping", "NsbasDeramping", "Ours" is shown in Figure 2a We can see that "NsbasDeramping" contains a quadratic phase ramp with respect to "EsaDeramping", that can reach perceptible values at the burst limits. "Ours" has the same phase content as "EsaDeramping". Therefore, the quadratic ramp is attributed to a slight imprecision in (9). As for Figure 2b, the azimuth spectrum at a certain range in the secondary burst has been computed after the deramping of method "Ours". We can see that there is spectral shift from the 0 frequency. We verified that the shift corresponds to 12 . We also verified that because of the burst synchronization property of Sentinel-1, and of typical values of quantities in (12), we can simplify the formula to

$$
f_{\text {shift }}=k_{t}(\tau) \eta_{l a g}+f_{\eta_{c}}(\tau) .
$$

We then predicted this spectral shift for all the bursts of the 31 secondary images in the dataset with 13 and the histogram is shown in Figure 3a. The maximal shift in absolute value is $\approx 35 \mathrm{~Hz}$. Again, this is due to the Sentinel-1 timing properties, and we should be able to safely assume that the spectral shift will be small in general for any dataset.

We also performed a test where (5) is modified into

$$
\begin{array}{r}
\phi\left(\eta, \tau, \eta_{\text {delay }}\right)=\exp \left\{-j \pi k_{t}(\tau)\left(\eta-\eta_{\text {ref }}(\tau)\right.\right. \\
\left.\left.-\eta_{\text {delay }}\right)^{2}-j 2 \pi f_{\eta c}(\tau)\left(\eta-\eta_{\text {ref }}(\tau)\right)\right\},
\end{array}
$$

with $\eta_{\text {delay }}$ a delay introduced in the de/re-ramping function such that the spectrum would be shifted by $k_{t} \eta_{\text {delay }}$. Resampling is then performed with a cubic spline. We can hence assess the effect of resampling a spectrally shifted burst on the

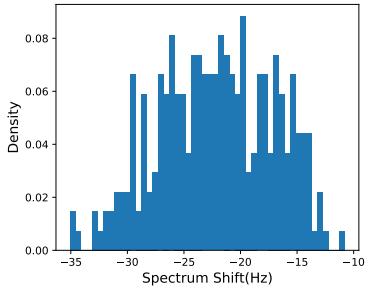

(a) Histogram of the distribution of the spectral shifts

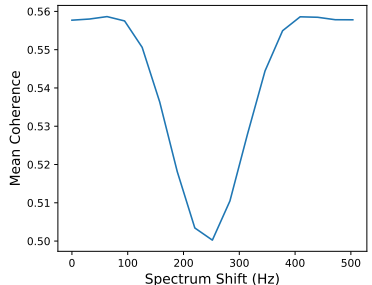

(b) The phase coherence vs simulated spectral shift
Fig. 3: The effect of spectral shift on the coherence. On the left, the distribution of the spectral shifts on the datastet. On the right, the degradation of the coherence after resampling the image with the spectral shift

interferometric phase coherence. Figure $3 \mathrm{~b}$ shows that the coherence doesn't drop until we reach high values of the spectral shift $(\approx 100 H z)$. Therefore, since the typical spectral shifts that we obtain with "Ours" should be small (for this dataset $\leq 35 \mathrm{~Hz}$ ), the resampling should not induce a coherence loss.

\section{CONCLUSION}

In conclusion, we have compared two deramping methods of the literature "EsaDeramping" [2] [3] and "NsbasDeramping" [1]. We introduced some improvements for the latter in "Ours". Those improvements were demonstrated experimentally on Sentinel-1 data. Nevertheless, the proposed method, like its predecessor in [1], only centers the spectrum to a certain degree and a small spectral shift will remain. Experiments showed that Sentinel-1 timing properties should limit the value of the residual spectral shift. Interpolating the signal should not induce a loss of interferometric phase coherence. Future work should examine the spatial structure of the phase error w.r.t. the spectral shift. Further studies should look into the safety of using this method, especially the effect of changing the interpolation kernel on the phase errors introduced.

\section{REFERENCES}

[1] Raphaël Grandin, "Interferometric processing of SLC Sentinel1 TOPS data," European Space Agency, (Special Publication) ESA SP, vol. SP-731, pp. 1-14, 2015.

[2] Nestor Yague-Martinez, Pau Prats-Iraola, Fernando Rodriguez Gonzalez, Ramon Brcic, Robert Shau, Dirk Geudtner, Michael Eineder, and Richard Bamler, "Interferometric Processing of Sentinel-1 TOPS Data," IEEE Transactions on Geoscience and Remote Sensing, vol. 54, no. 4, pp. 2220-2234, 2016.

[3] Nuno Miranda, "Definition of the TOPS SLC deramping function for products generated by the S-1 IPF," pp. 1-15, 2015. 